\title{
Journée du 15 novembre au Centre Georges
} Pompidou

Marie-Thérèse Jones-Davies (éd.)

\section{(2) OpenEdition}

\section{Journals}

\section{Édition électronique}

URL : http://journals.openedition.org/shakespeare/1112

DOI : 10.4000/shakespeare. 1112

ISSN : 2271-6424

\section{Éditeur}

Société Française Shakespeare

\section{Édition imprimée}

Date de publication : 1 novembre 1980

Pagination : 10-14

Référence électronique

"Journée du 15 novembre au Centre Georges Pompidou », Actes des congrès de la Société française Shakespeare [En ligne], 1 | 1980, mis en ligne le 01 novembre 2007, consulté le 30 avril 2019. URL : http://journals.openedition.org/shakespeare/1112; DOI : 10.4000/shakespeare.1112 


\section{SOCIÉTÉ FRANÇAISE SHAKESPEARE}

\section{ACTES DU CONGRĖS 1979}

DiRecteur DE LA PUblication

M.T. Jones - Davies 


\section{Le 15 Novembre}

\section{Pouvoir et sexualité : Mesure pour Mesure.}

Michel Grivelet introduisit la pièce, et le thème :

Pouvoir et sexualité sont, à l'évidence, les deux termes majeurs de l'argumentation dramatique dans Measure for Measure : c'est pour qưu'il vienne à bout de la licence générale des mœurs, en appliquant les lois dans toute leur rigueur, que le duc Vincentio délègue à Angelo son autorité;c'est parce qu'il est investi de cette autorité qu'Angelo rencontre Isabelle, la désire, et sé sert du pouvoir qu'il détient pour la contraindre à lui céder, pensant commettre impunément la faute pour laquelle il a condamné Claudio à mort.

Toutefois, aussi grand que soit l'intérêt qui s'attache à une analyse sans complaisance de l'appétit charnel, de sa violence, comme des subtilités d'une législation du mariage visant à en contenir les excès, la sexualité n'a dans la pièce qu'une importance relative. Elle sert de point d'application à une réflexion qui est avant tout réflexion sur l'exercice du pouvoir, selon ce qui est indiqué dès le premier vers :

"Of government, the properties to unfold »...

En prenant soin dès le début - même dans les échanges passionnés entre Angelo et Isabelle - d isoler le désir de tout sentiment d'une relation personnelle, Shakespeare en a délibérément restreint la portée dramatique. Au reste, une fois Angelo démasqué, on ne se soucie guère de ce qu'il adviendra d'une politique qui se proposait d'en finir avec la prostitution, le proxénétisme et la débauche. L'immoralité a cessé apparemment d'être un sujet brûlant. Elle est sortie, en tout cas, du champ de la pensée.

La différence, assez considérable, qui sépare les mœurs de notre temps de celles du temps de Shakespeare n'enlève rien à l'actualité de l'œuvre. La chasteté, sans doute, est démodée. Et Isabelle ne serait aujourd'hui qu'une curiosité si tout son rôle se ramenait, comme certains l'imaginent, à préférer sa virginité à la vie de son frère. Mais, vierge ou pas, le véritable enjeu est de savoir si la novice cèdera à un abus de pouvoir manifeste. Dans son livre récent sur la pièce, Rosalind Miles a tenu à citer les cas contemporains de quelques phallocrates (pour le coup c'est le mot qui convient) qui ont exercé sur des femmes la même sorte de chantage. La comédie n'a pas cessé d'être moderne, tant s'en faut. Son thème, c'est celui de l'attitude à observer envers le pouvoir, que l'on ait à l'exercer ou à le subir.

D'où la place faite aux rôles d'Angelo et de Vincentio. Tout le monde reconnaît la force dramatique du premier. Mais il en va autrement du second qui a été longtemps, et qui reste, controversé. Il a beau être à peu près le triple de celui d'Angelo et représenter, en nombre de mots, presque le tiers du texte total, il n'est pas rare qu'on le tienne pour accessoire et inconsistant. $\mathrm{Ou}$ bien il provoque une sorte de réprobation et d'animosité moralisatrices. Il est vrai que ces attitudes sont principalement le fait de critiques « littéraires ». La lecture se prête difficilement à l'appréciation des ressources scéniques et, d'une manière générale, elle n'est guère faite pour rendre justice à une pièce aussi délibérément théâtrale que Measure for Measure. L'épreuve de la mise en scène - Peter Brook 1'a encore montré, il y a peu, aux Bouffes 
du Nord - invite à en juger autrement. Elle porte, pour que le rôle soit véritablement tenu, à lui restituer, dans sa plénitude, la spécificité de sa fonction dramatique. La force des scènes comiques où Vincentio est ridiculisé par le persiflage de Lucio ou par la malice des événements et la stupidité intraitable de Bernardin, est en rapport direct avec l'argument principal de la pièce. En proie aux difficultés d'une situation qu'il a créée lui-même, réduit à l'impuissance par son propre stratagème et contraint d'avoir recours aux moyens les plus hasardeux pour éviter le pire, le duc est, plus que tout autre, en butte à l'ironie du pouvoir. C'est une chose bien dérisoire en politique que la passion de bien faire. La réflexion amorcée dès le début sur «l'art de gouverner 》 ne serait pas achevée sans Vincentio.

Cet exposé fut suivi d'une illustration scénique par Michael Kustow avec pour interprêtes Suzan Fleetwood et Michael Pennington. Puis d'un entretien avec François Marthouret et Maurice Benichou, interprêtes des rôles du Duc et de Lucio dans la mise en scène de Peter Brook, et avec Jean-Claude Carrière, auteur de l'adaptation française pour cette mise en scène.

\section{Les Femmes dans Shakespeare}

Le même jour une séance fut consacrée à ce thème, que présenta MarieThérèse Jones-Davies :

Bernard Shaw écrivait : "Chez Shakespeare, c'est la femme qui prend l'initiative 》, tandis que Virginia Woolf remarquait : "il serait impossible de dire ce que Shakespeare pensait des femmes ». Certes, il ne faut pas confondre l'art et la réalité et ne pas se demander avec A.C. Bradley quelle avait đû être l'enfance de Cordelia ou pour reprendre les termes de L.C. Knights « combien d'enfants eut Lady Macbeth ». L'arrière-plan historique et social a toutefois son importance. Les voyageurs étrangers qui visitent $1^{\prime}$ Angleterre élisabéthaine - tel Frederick, duc de Wirtemberg - notent que les femmes y sont plus libres qu'ailleurs et savent bien se servir de leur liberté. L'Angleterre passe pour être «le paradis des femmes mariées ». La bourgeoise de Londres est très décidée à améliorer son statut légal et Thomas Dekker explique, dans l'une de ses comédies, qu'une épouse à qui son mari a accordé pour un pouce de liberté «en prendra long comme une aune pour le moins».

$\mathrm{Au}$ XVIe siècle, le rôle de la femme paraît décisif dans la vie de la nation : les épouses d'Henry VIII contribuèrent à la séparation d'avec Rome; Marie Tudor engagea la contre-Réforme; Marie Stuart fut la première victime des luttes religieuses dans le conflit avec Elizabeth; enfin Elizabeth - Gloriana resta au pouvoir pendant quarante-cinq ans. Depuis la diatribe contre les femmes d'Edw. Gosynghill de 1541, les controverses allaient bon train à leur sujet. L'image de la femme idéale pour l'homme était soit la dame de Pétrarque soit la patiente Griselda, silencieuse et soumise. On connaissait les propos du philosophe Agrippa sur le refus illogique d'éduquer les femmes : "une femme dès sa naissance est maintenue dans l'oisiveté :... on ne lui permet de penser à rien qu'à son aiguille... quand elle atteint la maturité elle est abandonnée au gouvernement jaloux d'un mari ou enfermée dans le Bridewell des nonnes ». Dans La Première Sonnerie de Trompette contre le monstrueux 
gouvernement des Femmes, John Knox s'indignait qu'une femme (Marie Tudor) pût régner sur les hommes contre la volonté de Dieu, contre l'équité et la justice, puisque la nature a fait les femmes faibles, impatientes, inconstantes. Son livre sortit de presse en 1558, l'année où Elisabeth accédait au trône; il eut de la peine à se faire pardonner. Plus tard, en 1615, Le Procès des Femmes, par Joseph Swetnam, suivi de leur louange, est empreint de misogynie tout en faisant deviner que la femme est un mal nécessaire. Malgré tout, avec la montée de la classe bourgeoise, la femme devient socialement respectable. Les humanistes (ex. Thomas More) se sont élevés contre l'amour courtois; leur influence se fait sentir. Si toutefois Francis Bacon dans son essai sur « Le mariage et le célibat 》 révèle sa philosophie utilitaire : "L'épouse est une maîtresse pour l'homme jeune, une compagne pour l'âge mûr, une garde-malade pour le vieillard », John Donne offre un point de vue bien différent quand il montre que "déifier la femme est impie; en faire un démon est diabolique, en faire une maîtresse est indigne d'un homme, en faire une servante n'est pas noble, mais en faire une épouse, voilà une attitude à la fois sainte et virile ».

Les diverses idées qui avaient cours en son temps se retrouvent chez Shakespeare à divers degrés, plus ou moins nuancées ou mises en question.

Le double legs du mythe de l'amour courtois et du scepticisme humaniste devant ce mythe réapparaît dans Peines d'Amour Perdues : les jeunes gens de Navarre courtisent des divinités sans reconnaître dans les Dames venues de France des êtres raisonnables. Les Dames, elles, acceptent cette cour comme un simple intermède de comédie. Shakespeare complique et approfondit par l'ironie les images toutes faites des relations de l'homme et de la femme. L'ironie de Hamlet n'est jamais plus mordante que lorsque, faisant allusion à «l'union mystique» du mariage, il appelle Claudius «ma chère mère ». A Claudius qui objecte : " ton père ", il répond : " ma mère - père et mère est époux et épouse, époux et épouse ne sont qu'une seule chair, et ainsi vous êtes ma mère ». Malgré cette ironie, il ne rejette pas la croyance à l'indivisibilité de l'époux et de l'épouse, sans quoi il pourrait justifier l'infidélité de Gertrude et son remariage. Hamlet, devenu misogyne se fait acteur dans sa relation avec Ophélie pour obscurcir par la satire généralisante (Fragilité, ton nom est fe mme) l'image de la femme individuelle. Quant à Ophélie, elle est l'exemple de la femme conditionnée par l'éducation reçue; privée de la tutelle de Polonius, elle n'a a ucune identité et sombre dans la folie.

Que les rôles de femmes soient tenus par de jeunes garçons déguisés en femme pose le problème de l'homosexualité, mais ce problème reste beaucoup plus ambigu que dans une pièce élisabéthaine comme Edouard II de Marlowe.

Par le jeu du déguisement en homme, la femme gagne son indépendance. Pour pouvoir agir, elle doit être respectée; par le déguisement, sa féminité reprend tous ses droits, féminité plus réelle et plus durable que celle seulement symbolisée par le vêtement. Les droits de la femme passent par le jeu pour être reconnus (Viola, Rosalinde). Le double rôle d'homme et de femme qu'adopte successivement Rosalinde-Ganymède dans la forêt met en relief la nature théâtrale à la fois de la masculinité et de la féminité. Le jeu auquel se prête Orlando en acceptant de prétendre que Ganymède est Rosalinde réprime en lui toute tentation à l'exagération lorsqu'il essaie de convaincre « Rosalinde » 
de son amour. L'ironie du déguisement supprime ce qu'il y a d'artificiel dans la relation homme-femme, telle qu'elle apparait dans le couple Phébé-Silvius.

Le comble du déguisement est celui d'Hermione, qui se transforme en statue pour faire émerger ce qu'elle est. Juliette devra passer par la mort. Même Cléopâtre fait dire à Antoine qu'elle est morte. Le déguisement du mensonge luj permettra de reconquérir Antoine et de redevenir reine. En fait, Shakespeare ne cherche pas à opposer la femme à l'homme, à diviser la nature humaine en " masculine » et "féminine ». Il semble plutôt refuser de séparer physiquement, intellectuellement et spirituellement le monde des hommes de celui des femmes.

Portia (Marchand de Venise) se transforme en homme de loi pour obtenir justice; mais le pièce montre qu'elle n'aura droit à sa féminité que si l'homme (Antonio, Bassanio) a le droit d'être libre. Dans La Tempête également, Miranda ne peut être elle-même tant que Ferdinand reste esclave; comme si les femmes ne pouvaient exister vraiment que dans une société où les hommes sont libres.

Outre le jeu du déguisement qui peut être toute patience (Hermione), il y a le jeu de l'éloquence.

Dans la pensée populaire, la parole est un talent dont abusaient les femmes. C'est que la parole donne la puissance. La vérité est silencieuse (Cordelia), et pourtant Cordelia est bafouée; si Coriolan appelle Virgilia " mon gracieux silence », c'est Volumnia qui l'influence. Dans les comédies, les femmes «qui sauvent », Beatrice, Rosalinde, ont une éloquence éblouissante, elles jouissent d'une merveilleuse fantaisie libératrice qui tient de la magie : une étoile dansait quand naquit Beatrice. Portia (Marchand de Venise:) comprend l'efficacité de l'éloquence quand elle se fait avocat. C'est le jeu que devra s'efforcer de jouer Isabelle (Measure for Measure). Selon Claudio, elle possède l'art de savoir bien convaincre. Cependant au début elle parle peu, elle échoue et doit être encouragée par Lucio. Kate (La Mégère Apprivoisée), on pourrait dire, est comme la satire de l'idéal que l'homme se fait de la femme. Par l'intelligence, et à la fin par l'éloquence elle entre dans le jeu de Petruchio. Elle doit pour gagner jouer le jeu de la rhétorique : c'est la victoire de l'esprit (wit) qui permet aux hommes et aux femmes de se comprendre. Dans Othello, Desd émone est à l'origine vive et éloquente : elle sait convaincre les Sénateurs. Une fois accusée par le Maure, elle perd son éloquence, car le langage que lui tient Othello n'a plus pour elle de réalité. C'est pour sauver l'idéal de la perfection féminine auquel il croit qu'Othello tue la femme réelle qui possédait l'intelligence et l'esprit. L'ironie qui pénètre la fin de la tragédie vient de ce que Shakespeare donne à Emilia, la volubile, le pouvoir de sauver la vérité de la vraie Desd émone.

Dans la tragédie shakespearienne, il y a aussi la femme rendue dangereuse par son impuissance à "agir » dans le monde masculin de l'action. N'agissant pas elle-même, elle devient par compensation instigatrice de l'action. Lady Macbeth agit sur l'image de l'homme que se fait Macbeth. Pour pousser Macbeth à l'action, elle assume la virilité. Mais sa fiction de masculinité n'est que paroles. Elle ne peut complètement réprimer sa nature : "Je l'aurais fait moimême, s'il n'avait ressemblé à mon père endormi. »(II.ii). La violence détruit Lady Macbeth qui connaît la plus grande solitude dans un monde où la femme est dépendante de l'homme. On constate que si Lady Macbeth assume la virilité 
de Macbeth, Macbeth, lui, devient « une petite fille »(III.iv.106). La même chose se produit dans Le Roi Lear, où la folie peut exprimer le passage d'un sexe à l'autre. Lear souffre de l'hysterica passio, qui est une maladie de la femme. Ce symbolisme n'est pas très éloigné de ce qui apparait aussi dans Antoine et Cléopâtre. Enfin la femme violente, comme Regan, Goneril, n'est pas sans évoquer la Méd ée de Sénèque.

On pourrait distinguer beaucoup d'autres aspects de la femme et de son rôle dans Shakespeare, où elle apparaît tentatrice ou prophétesse, séductrice ou protectrice, dans toute son infinie variété.

Après cet exposé une scène de La Tempête fut présentée par Patrick Baty avec J.P. Belfort, Christine Catala et Hélène Hamon. Elle fut suivie d'un débat sur Comme il vous plaira, animé par Mme Jones-Davies, et auquel participèrent Simone Benmussa, Beno Besson, et Coline Serreau, interprête de Rosalinde dans la mise en scène de celui-ci.

\section{Le 18 Novembre}

\section{L'Homme nu \\ Les Fous et la folie dans Shakespeare}

Ce thème fut présenté par Jean Fuzier:

Nous distinguerons d'emblée, comme le faisaient Shakespeare et des contemporains, et comme nous y invite le programme de cette journée, qui associe Comme il vous plaira et Le Roi Lear, entre le "fool 》 et le «madman 》, le simple d'esprit (parfois aussi bouffon) et le dément authentique. L'un et l'autre avait d'ailleurs sa place à l'asile, mais l'un et l'autre participait aussi d'une sagesse supérieure, à la mesure de ce qui était, aux yeux des hommes raisonnables, sa folie. Fou de Comme il vous plaira,Touchstone-pierre de touche de quoi, sinon du bon sens ? - folie à trois de Lear, recréant, avec son authentique dément (le roi), son simple d'esprit promu au rang de fou professionnel (le bouffon), et son simulateur cherchant sous le masque de la folie une protection immédiate autant que les moyens d'une vengeance ultérieure (Edgar), la structure d'un asile de theâtre mis à la mode quelques mois plus tôt par The Honest Whore de Dekker et Northward Ho ! de Dekker et Webster, sous la double influence, satirico-morale et socio-historique, des " hôpitaux 》 sy mboliques (Wireker, Lydgate, Brant, Erasme, Balsac, Copland) et de l'observation réaliste de l'hôpital des fous, concrétisée par L'Hospidale de' pazzi incurabili de Garzoni (1586), véritable détonateur européen de l'asile au théâtre, qu'il s'agît de l'Espagne, de l'Angleterre ou de la France, ce sont là les deux aspects extrêmes du problème : du plus simple et du plus immédiat, encore qu'il englobe toute la tradition élisabéthaine du bouffon (dont celui de Lear sera l'ultime et le plus grand), au plus riche et au plus complexe, puisque c'est toute une tradition internationale, issue du Moyen Age et modelée d'un pays à l'autre par l'observation de la condition spécifique des aliénés, qui s'exprime finalement dans Le Roi Lear. 